\title{
NODAL SIMULATION OF SUSPENDED MEMS WITH MULTIPLE DEGREES OF FREEDOM
}

\author{
Jan E. Vandemeer, Michael S. Kranz, and Gary K. Fedder * \\ Department of Electrical and Computer Engineering, and ${ }^{*}$ The Robotics Institute \\ Carnegie Mellon University, Pittsburgh, Pennsylvania 15213-3890 \\ Telephone: (412) 268-3767Ｆax: (412) 268 - $2860 \quad$ E-mail: jev@prowler.ece.cmu.edu
}

\begin{abstract}
A mixed-domain schematic representation compatible with nodebased behavioral simulators is developed for rapid design of suspended microelectromechanical systems (MEMS). Hierarchical models of several basic microelectromechanical elements are created. The components are interconnected to represent both devices and more complex systems. Using beams, masses, and electrostatic actuators as the fundamental building blocks, a nodal simulation of complete in-plane motion of a crab-leg resonator demonstrates the design approach. Electrical properties are included in the component models, enabling electrical and electromechanical signal analysis to be performed. This work is part of an continuing project that eventually will provide full six DOF models for a broad class of MEMS.
\end{abstract}

\section{INTRODUCTION}

The increasing integration of microelectromechanical systems (MEMS) has pushed the demand for computer-aided design (CAD) tools to support rapid design of systems involving physical interactions between electrical, mechanical, electrostatic, magnetic, thermal, fluidic, and optical domains. For standard integrated circuits, design is eased by abstracting physical layout into a schematic view which represents the circuit as an interconnected set of components. We describe continuing work on a MEMS schematic view and an associated mixed-domain nodal simulation which incorporates a hierarchical library of MEM component models.

At present, MEMS simulation is most often performed using direct numerical simulation (e.g., finite element analysis), or signal flow analysis. Finite element analysis is arduous and time consuming for system design due to its low level of abstraction, and lack of design hierarchy. Previous work on higher-level MEMS structural design has focused on behavioral simulation of individual devices (e.g., microresonators) with abstract macromodels [1], or with eigenmode decomposition using single degree-of-freedom (DOF) elements [2][3].
In contrast, nodal simulation enables a structured representation for MEMS design using a hierarchical set of MEM components, which can be interconnected in a general way to create more complicated components and systems. The resulting schematic view provides a direct linkage between both physical layout and behavioral simulation, as is the case with standard integrated-circuit design. A key feature is the one-toone correspondence of components to layout, which provides an intuitive interface for the designer. Coupling the schematic methodology with existing schematic capture tools that are compatible with electrical circuit analysis enables MEMS design to be quick and efficient.

Our initial implementation is restricted to surface-micromachined suspended MEMS. Suspended MEMS have no unconstrained mechanical elements, and include such devices as resonators, accelerometers, and vibratory-rate gyroscopes. Basic building-block components to construct these devices include beam springs, plate masses, and electrostatic gaps.

The next section describes the basic concepts of the MEMS schematic and nodal simulation, including component descriptions and model implementations in an analog hardware description language (HDL). An example schematic design, a crab-leg flexure microresonator, is constructed using several basic building-block components. A sampling of transient and frequency-domain simulations of the crab-leg resonator demonstrate the utility of the schematic design method.

\section{THEORETICAL}

\section{Variable Conventions}

Static equilibrium dictates that the forces acting on a body must sum to zero. Likewise the sum of moments must also equal zero. These equations are the "through" variable relations, and are analogous to Kirchhoff's Current Law in circuit theory. Nodal simulators solve for system variables by making the sum of the "through variables" flowing 

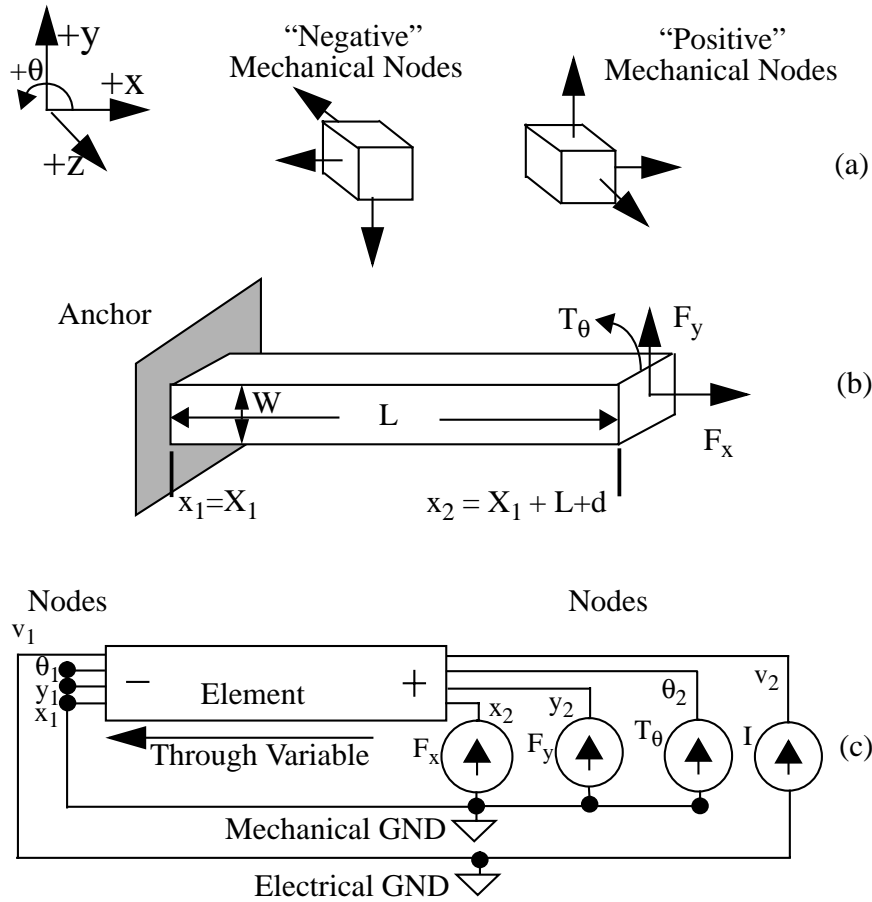

Fig. 1. (a) Conventions for positive nodes on a mechanical element, (b) Mechanical Element with Axial tensile force, $F_{x}$, transverse force $F_{y}$, and bending torque $T_{q \cdot}$ (c) Equivalent schematic.

out of each node equal to zero. By defining component models (templates) using an Analog Hardware Description Language (HDL), one can create equations which relate the multi-domain through variables in terms of the across variables. The simulator solves for the across variables within each template, and then solves equations involving through variables via substitution.

The present discussion is restricted to simulation of in-plane motion $(x, y, \theta)$, with coupled electrostatic and electrical effects. Therefore in our current formulation, force in $\mathrm{x}$ and $\mathrm{y}$, moment about $\theta$, and current are through variables, while $\mathrm{x}, \mathrm{y}$, and $\theta$ and voltage are across variables.

In contrast to conventional electrical schematic components, MEMS components have a certain physical size and orientation associated with them (e.g., A beam flexure has a length, a plate mass has a length and width). These user-defined values are incorporated into the components. For example, when a horizontal beam is placed into a schematic, its left (or minus) side has a certain position ( $x, y, \theta)$, its right (or plus) side has the position $(\mathrm{x}+$ length, $\mathrm{y}, \theta)$. This is the same for a vertical beam, except the displacement goes from bottom to top (+y direction).

The convention used by nodal simulators is that when a through variable flows from the positive node to the negative node it is considered to have a positive value. Mechanical nodes associated with surfaces whose normal is directed along the $+\mathrm{x},+\mathrm{y}$, or $+\mathrm{z}$ axes are considered positive (Fig. 1(a)). We define a positive through force flowing into a node as equivalent to the actual force directed along the $+x$ or $+y$ axes.
Similarly, a through moment flowing into a node is equivalent to a counterclockwise torque around the $+\mathrm{z}$ axis (right-hand rule). For example, Fig. 1 illustrates the schematic representation of a mechanical element subjected to an axial force $F_{x}$, a transverse force $F_{y}$, a bending torque $T_{q}$, and an electrical current, I

\section{Simple Beam Model}

A fundamental element in MEMS is the simple beam. The nodal simulation methodology is introduced by discussing the lumped model for a simple beam and deriving a relation between forces and positions. Most surface-micromachined suspensions are composed of one or more beams of this type.

A beam of length $L$ is shown in Fig. 2 with arbitrary orientation in the $x-y$ plane. The sign convention is chosen so that forces and moments are positive in the direction of the normals. Global coordinates are in upper case letters, and local coordinates along beams are in lower case letters.

For the beam of length $L$, the through variable relations are found from the simple geometry.

$$
\begin{gathered}
F_{a, x}=-F_{b, x} \\
F_{a, y}=-F_{b, y} \\
M_{a, \psi}=-M_{b, \psi}+L \sin \left(\theta_{a}\right) F_{b, x}-L \cos \left(\theta_{a}\right) F_{b, y}
\end{gathered}
$$

The through variable (i.e., the moment) in (3), is different from the electrical analogy in that its magnitude is not necessarily equal at the two beam nodes.

The beam bending is embedded in an analytic equation relating through to across variables. As a first approximation, we have modeled a massless, lossless, uniform beam with no axial stiffening effect. The beam displacement relation $y(x)$ obeys the differential equation where $E$ is Young's modulus, $I_{x y}$ is the beam bending moment of inertia the $x-y$ plane, and $q(x, t)$ is the distributed external force along the beam. External forces are assumed to be lumped at the nodes, so $q(x, t)=0$. There is

$$
\frac{\partial^{2}}{\partial x^{2}}\left(E I_{x y} \frac{\partial^{2}}{\partial x^{2}} y(x, t)\right)=q(x, t)
$$

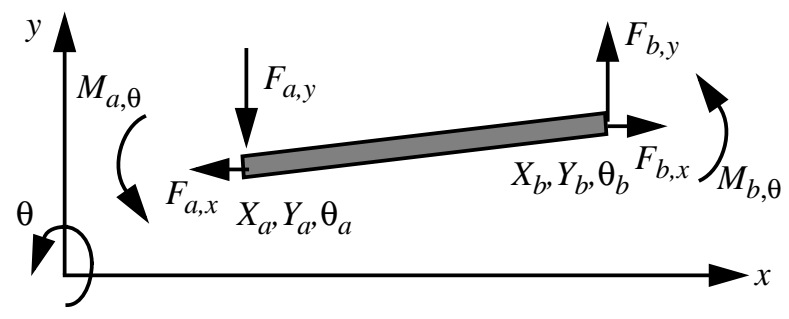

Fig. 2. Beam in global coordinate system, showing sign convention of forces and moments. The unloaded beam is of length $L$ from node $a$ to node $b$. 
no loss in generality if we work in a local coordinate system where node $a$ is a local origin. Rigid body translation and rotation of the beam can be included after determining the displacement due to bending. Using the boundary conditions, (4) is solved to find the relative displacement at node $b$.

$$
\left[\begin{array}{c}
x_{b} \\
y_{b} \\
\theta_{b}
\end{array}\right]=\left[\begin{array}{ccc}
\frac{L}{E A} & 0 & 0 \\
0 & \frac{L^{3}}{3 E I} & \frac{L^{2}}{2 E I} \\
0 & \frac{L^{2}}{2 E I} & \frac{L}{E I}
\end{array}\right]\left[\begin{array}{l}
f_{b, x} \\
f_{b, y} \\
m_{b, \theta}
\end{array}\right]
$$

which can be expressed more compactly as $\boldsymbol{x}_{b}=\boldsymbol{A} \boldsymbol{f}_{b}$, where $\boldsymbol{x}_{b}$ and $\boldsymbol{f}_{b}$ are generalized local displacement and force vectors, and $\boldsymbol{A}$ is the 3-DOF compliance matrix of the beam. The transformation from the global to local coordinate systems requires determination of the position of the local origin, $\boldsymbol{X}_{a}$, the rigid-body rotation matrix, $\boldsymbol{W}$, and the zero-force (at rest) position, $\boldsymbol{L}_{b}$, of $\boldsymbol{x}_{b}$ in the local frame.

$$
\boldsymbol{X}_{a}=\left[\begin{array}{c}
X_{a} \\
Y_{a} \\
\theta_{a}
\end{array}\right] ; \quad \boldsymbol{W}=\left[\begin{array}{ccc}
\cos \theta_{a} & -\sin \theta_{a} & 0 \\
\sin \theta_{a} & \cos \theta_{a} & 0 \\
0 & 0 & 1
\end{array}\right] ; \quad \boldsymbol{L}_{b}=\left[\begin{array}{l}
L \\
0 \\
0
\end{array}\right]
$$

Where $\boldsymbol{L}_{b}$ is given in (6) for the simple beam example. The position of node $b$ is

$$
\boldsymbol{X}_{b}=\boldsymbol{X}_{a}+\boldsymbol{W} \boldsymbol{L}_{b}+\boldsymbol{W} \boldsymbol{A} \boldsymbol{W}^{1} \boldsymbol{F}_{b}
$$

From a circuit simulation perspective, the beam element is analogous to a vector voltage source of value $\boldsymbol{W} \boldsymbol{L}_{b}$, in series with a resistance matrix of value $\boldsymbol{W A} \boldsymbol{W}^{\boldsymbol{1}}$. The equation relating the through variables, $\boldsymbol{F}_{b}$, to across variables is found by inverting (7).

$$
\boldsymbol{F}_{b}=\boldsymbol{W} \boldsymbol{K} \boldsymbol{W}^{-1}\left(\boldsymbol{X}_{b}-\boldsymbol{X}_{a}\right)-\boldsymbol{W} \boldsymbol{K} \boldsymbol{L}_{b}
$$

Where $K=A^{-1}$ is the stiffness matrix.

Thus far, the models for the beams have no mass. The mass of the beams can be approximated by placing an external mass element on the end of the beam. The development of a high quality effective mass model is presently being researched.

\section{SIMULATION RESULTS}

\section{Model Implementation}

Several basic building-block components have been created. A subset of the general beam model is presently implemented as two simple beam components, one with horizontal and one with vertical orientation. In order to connect two beams perpendicularly to one another, a joint component has been created. The joint accounts for the change in angle from a vertical beam to a horizontal beam.

Other models include a rectangular plate masses, an electrostatic comb-finger actuator, and a mechanical anchor. The rigid-body plate mass model includes translational and rotational inertia with air damping approximated as an equivalent Couette flow [4]. Electrostatic comb-finger actuators [5] are modeled with simple behavioral equations described in [6]. The actuators also serve as capacitive displacement sensors. Anchor components fix the global position of a node, analogous to a voltage source.

The derived through variable relations are implemented in an ana$\log$ HDL [7] template file for each element model. This file, when incorporated into a schematic, is read in by the simulator, resulting in the formation of the appropriate equivalent conductance matrix for solution.

\section{Crab-Leg Resonator Schematic Example}

A schematic of a symmetric crab-leg resonator using the basic components is shown in Fig. 3.

A $2 \mu \mathrm{m}$-thick polysilicon surface micromachined process is chosen to determine suitable element parameters. Each crab-leg consists of one $2 \mu \mathrm{m}$-thick by $2 \mu \mathrm{m}$-wide by $100 \mu \mathrm{m}$-long vertical beam, one $2 \mu \mathrm{m}$ -

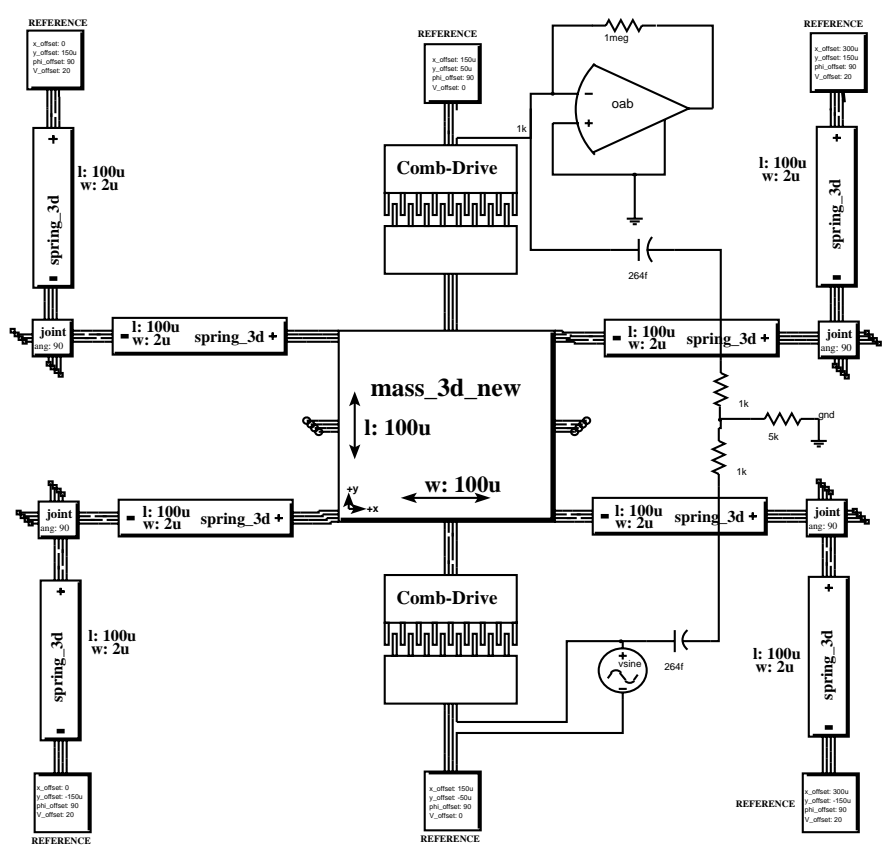

Fig. 3. Schematic of the crab-leg resonator. 
thick by $2 \mu \mathrm{m}$-wide by $100 \mu \mathrm{m}$-long horizontal beam, and a $90^{\circ}$ joint to connect the beams properly. The central mass is $100 \mu \mathrm{m}$ wide by $100 \mu \mathrm{m}$ long by $2 \mu \mathrm{m}$ thick. The electrostatic comb-drive actuator contains 15 fingers, each of length $15 \mu \mathrm{m}$, with a finger overlap of $5 \mu \mathrm{m}$, and gap of $2 \mu \mathrm{m}$.

A voltage of $20 \mathrm{~V}$ ac with a $20 \mathrm{~V}$ dc offset is applied to the actuators to provide the driving force needed to actuate the mass. The transresistance amplifier senses the variation in capacitance of the interdigitated comb capacitive sensor. Feedthrough capacitance, $\mathrm{C}_{\mathrm{f}}$, between the substrate and the comb drives, and substrate resistance, $\mathrm{R}_{\text {sub }}$, are incorporated in the schematic to determine their effect on the output voltage signal. Eventually, these parasitics may be added to the anchor model.

The resonator schematic was simulated using a commercial mixeddomain behavioral simulator [8]. The rotational and translational displacements at the mid-point of the mass were examined, as well as the electrical signal coming from the transresistance amplifier.

To compare the analytically derived value for the displacement to the simulated value, a DC analysis was performed at $+20 \mathrm{~V}$ and $-20 \mathrm{~V}$ and, the $y$-position of the center of mass was recorded. The difference in displacement of the center of mass came out to be $32.2 \mathrm{~nm}$. This value matched the analytically derived value of $32.2 \mathrm{~nm}$.

First, an ac sweep was performed to determine the resonant frequency in the $y$ direction (Fig. 4). The simulated value of resonant frequency is $59.94 \mathrm{kHz}$, which is within $1 \%$ of the analytical value of $59.89 \mathrm{kHz}$. It was also found that if the accuracy of the simulation was increased, and the range over which the analysis was performed was narrowed, that the resonant frequency arrived at through simulation matched exactly with the analytical results.

A group of transient analyses at varying frequencies was performed to determine the center of mass' displacement in the $y$ direction at fre-

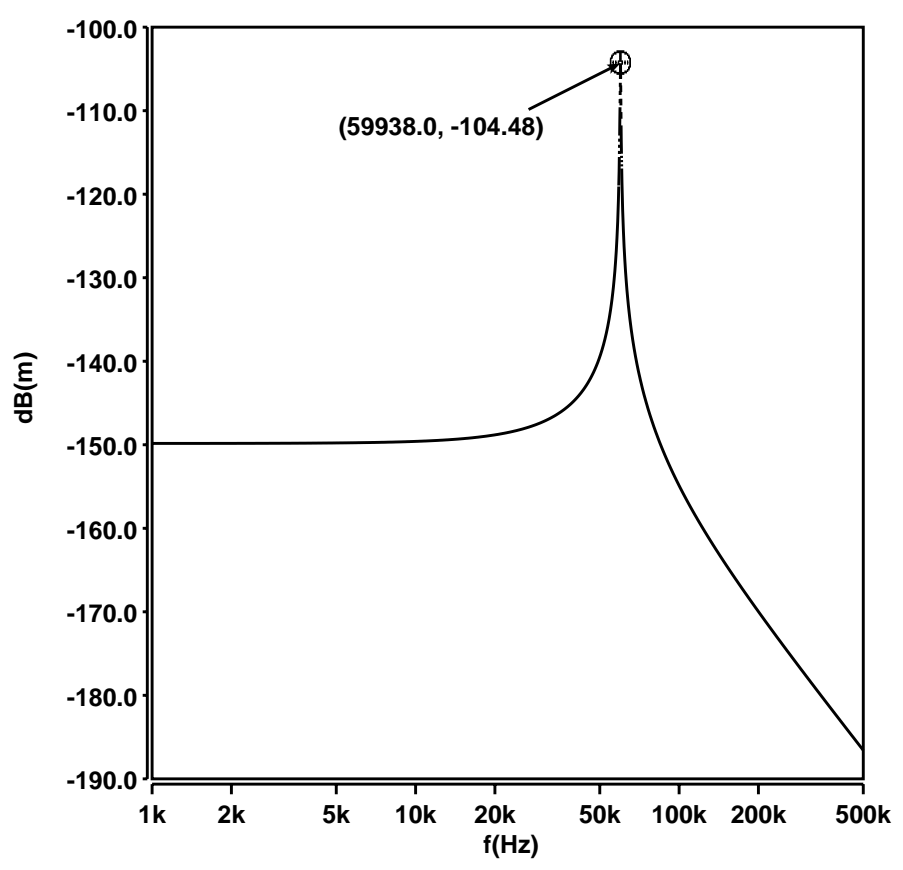

Fig. 4. AC analysis of crab-leg resonators center of mass displacement in the $y$ direction.

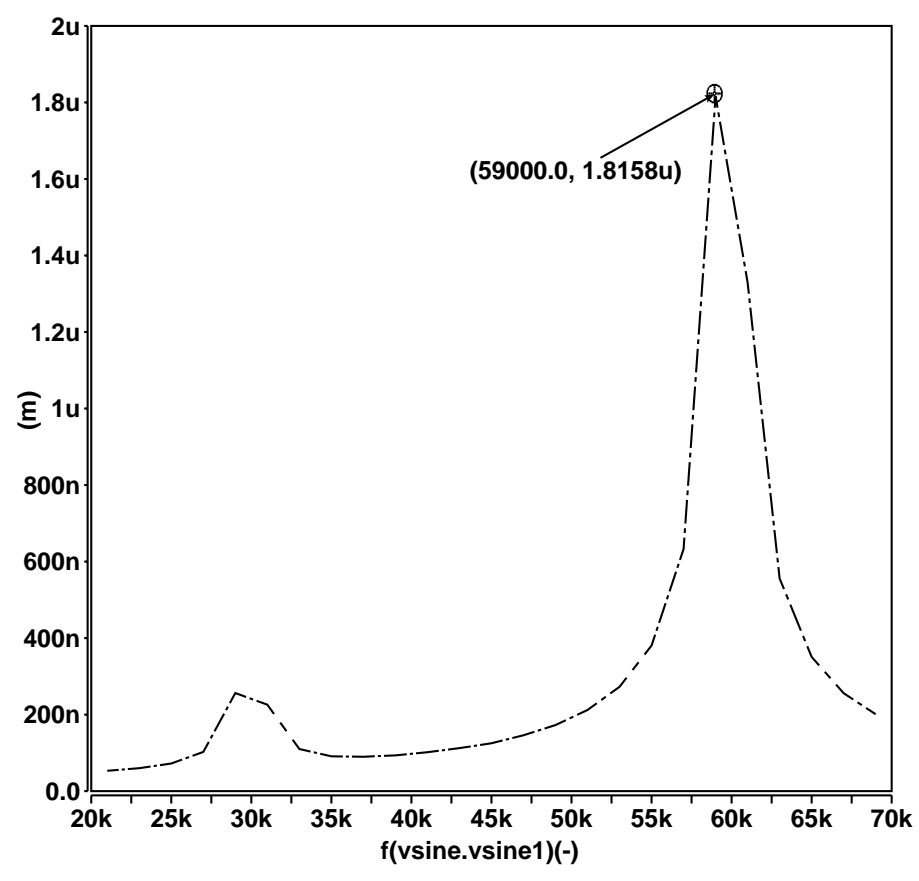

Fig. 5. Maximum y-displacement of the center of mass at various frequencies

quencies that neighbor resonance. Peak-to-peak displacement at each frequency is plotted as a function of frequency in Fig. 5. In the group of transient analyses, the ac frequency of the comb-drive voltage was stepped from $21 \mathrm{kHz}$ to $69 \mathrm{kHz}$ by increments of $2 \mathrm{kHz}$ to include both the fundamental and second harmonic excitation of resonance. Each analysis was run for $500 \mu$ s which yielded results of approximately $30 \%$ its steady state value. The primary resonant peak occurred at $59 \mathrm{kHz}$. The second peak at half the resonant frequency is the $\mathrm{V}^{2}$ nonlinearity of the comb drive. This effect is not detected by the ac simulation in Fig. 4. since it linearizes about a dc operating point.

A transient analysis performed at the resonant frequency is plotted in Fig. 6. The center of mass' vertical displacement and velocity, and the output voltage from the transresistance amplifier are plotted as a function of time. This waveform displays the steady state response of the resonator. The y-displacement has a peak-to-peak value of $6.24 \mu \mathrm{m}$, giving a Q factor of 194, which is within $2 \%$ of the analytically calculated value of 197. It was also found that the lower the maximum step size in the simulation, the closer the $\mathrm{Q}$ factor came to the analytical result. The output voltage is determined by the current flowing through the upper comb drive, which puts the output voltage signal in $180^{\circ}$ phase with the plate velocity.

An additional transient analysis was performed with the schematic was slightly modified to induce the comb-fingers to crash into one another. This was accomplished by altering the $x$ offset on the position references connected to the stator comb fingers by $-0.1 \mu \mathrm{m}$ (to the left). This created unequal gaps on either side of each comb finger, thereby providing a small destabilizing $\mathrm{x}$-directed force. A transient analysis was performed with the pulsed drive voltage profile shown in Fig. 7(a). The electrostatic force that attracts the comb-fingers is proportional to the 


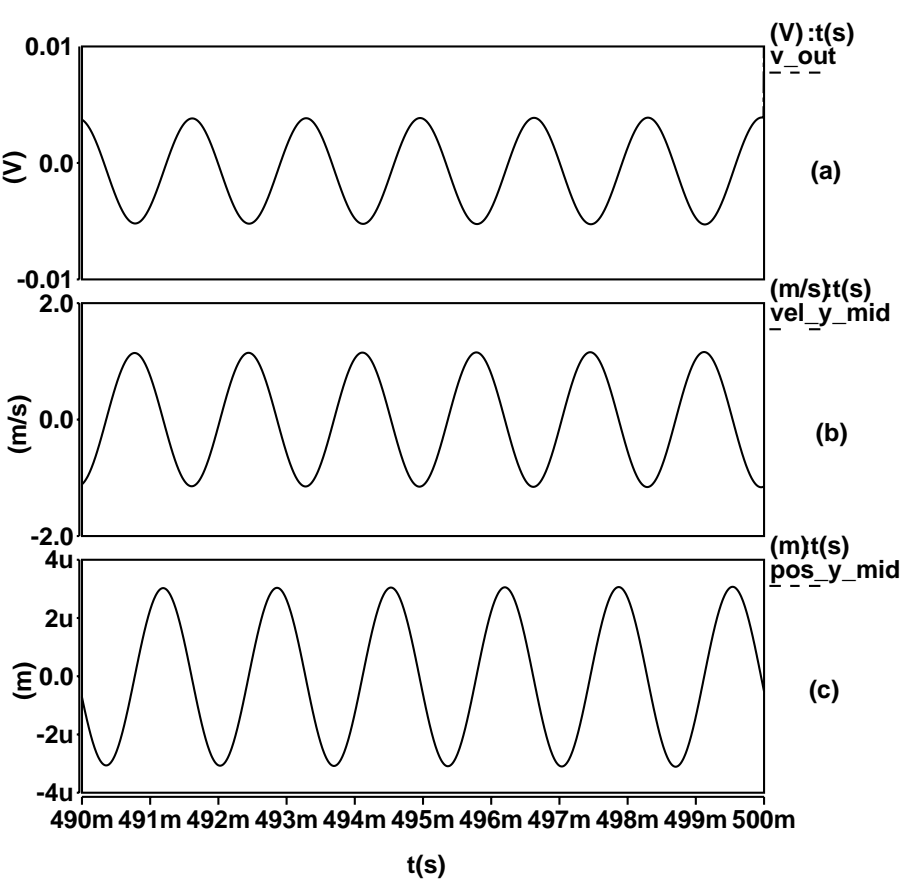

Fig. 6. Transient waveform of the (a) output voltage, (b) velocity (in y) of the center of mass and (c) position of the center of mass ( $y$ direction).

square of the voltage between the fingers. An instability is reached when the electrostatic force becomes equal to the restoring force provided by the flexure. At this point the fingers crash into each other (Fig. 7(a)). In our simulation we assume that the fingers have a native oxide layer to prevent shorting. The simulated value of the finger to finger threshold voltage was $80.3 \mathrm{~V}$. This is within $5 \%$ of the analytical value of $76.7 \mathrm{~V}$. Once the fingers have crashed, the voltage across the fingers is ramped down. Again, we cross a threshold when the electrostatic force decreases enough to equal the forces provided by the flexure. The analytical value for the releasing threshold voltage in our system is $1.0 \mathrm{~V}$, the simulated value is $0.98 \mathrm{~V}$. Once the fingers have released, the system begins oscillating due to inertia. The system oscillates at the resonant frequency of the system $(59.89 \mathrm{kHz})$. The oscillation decays, and eventually ends due to the damping factor.

\section{CONCLUSION}

A schematic design method of analyzing various MEMS, has been demonstrated. The MEMS nodal simulation is compatible with conventional circuit simulation, and can readily be implemented in tools which support analog HDL. The MEMS schematic can be altered quickly to perform experiments, and iterative design. The example schematic of a crab-leg resonator was constructed from elementary components, and successfully analyzed using a simulator that simulates the electrical, mechanical, and electromechanical characteristics of the device.

For resonant MEMS structures involving small angle deflections and reasonable proportions between the beams and masses, these current element models provide quick and accurate solutions. Simulations will

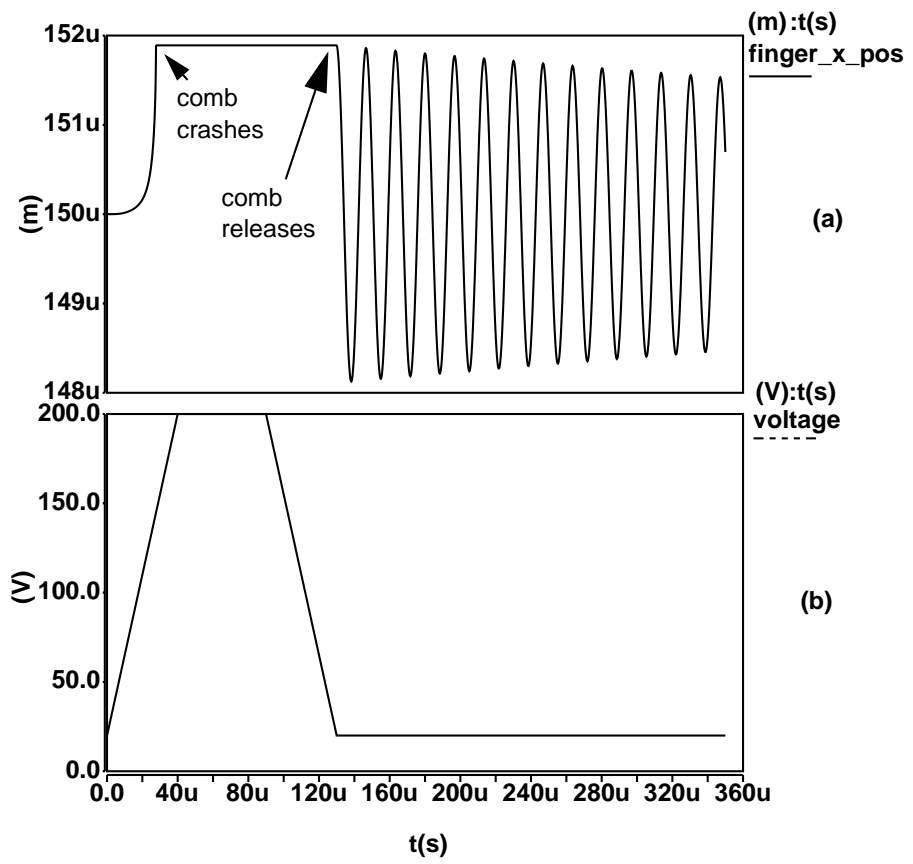

Fig. 7. Transient waveform of comb-finger crashing. (a) Voltage pulse. (b) Comb-fingers motion in $\mathrm{x}$.

lose some accuracy for resonant structures containing stiff beams and a large plate mass. These situations require the plate's compliance to be taken into consideration for an accurate simulation.

In the future, advances are expected to be made in component models accuracy, and increased degrees of freedom (eventually full 3D dynamics). In addition, more components will be created, including lumped parameter models of various surface micromachined suspensions (i.e., folded-flexure, crab-leg).

\section{ACKNOWLEDGEMENTS}

The research effort is sponsored by the Defense Advanced Research Projects Agency under Rome Laboratory, Air Force Materiel Command, USAF, under grant number F30602-96-2-0304 and in part by G.K.Fedder's National Science Foundation CAREER Award MIP9625471.

\section{REFERENCES}

[1] Karam, J.M., Courtois, B., Hofmann, K., Poppe, A., Rencz, M., Glesner, M., and Szekely, V., January, 1996, "Micro-systems Modeling at a System Level”, $A P C H D L$ '96, Bangalore, India.

[2] Berg, E. C., Lo, N. R., Simon, J. N., Lee, H. J., and Pister, K. S. J., April 1996, "Synthesis and Simulation for MEMS Design", ACM SIGDA Physical Design Workshop, Reston VA, pp. 66-70.

[3] Scholliers, J., and Yli-Pietila, T., May 1995, "Simulation of Mechatronic Systems Using Analog Circuit Simulation Tools", Proc. IEEE Intl. Conference on Robotics and Automation, Nagoya, Japan, vol. 3, pp.2847-52. 
[4] Zhang, X., and Tang, W. C., 1995, "Viscous Air Damping in Laterally Driven Microresonators," Sensors and Materials, v. 7, no. 6, pp.415430.

[5] Tang, W. C., Nguyen, T. C. H., Judy, M. W., and Howe, R. T., February 1990, "Electrostatic Comb Drive of Lateral Polysilicon Resonators," Sensors and Actuators A, vol. 21, no. 1-3, pp. 328-31.

[6] Johnson, W. A., and Warne, L. K., March 1995, "Electrophysics of Micromechanical Comb Actuators," J. of Microelectromechanical Systems, v.4, no.1, pp.49-59.

[7] MAST Reference Manual, Release 4.0, Analogy Inc., Beaverton OR, 1995.

[8] SaberGuide and SaberScope Manual, Release 4.0, Analogy Inc., Beaverton OR, 1995. 